\title{
Modeling of altered layer formation during reactive ion etching of GaAs
}

\author{
A. Mutzke ${ }^{a},{ }^{*}$ A. Rai ${ }^{a}$, R. Schneider ${ }^{b}$, Angelin E. J. ${ }^{b}$, R. Hippler ${ }^{b}$ \\ ${ }^{a}$ Max-Planck-Institute of Plasmaphysics, EURATOM Association, D-17491 Greifswald, \\ Germany \\ ${ }^{b}$ Institute of Physics, Ernst-Moritz-Arndt-University Greifswald, Felix-Hausdorff-Str.6, \\ D-17489 Greifswald, Germany
}

\begin{abstract}
The binary collision based SDTrimSP model has been used to simulate the reactive ion beam etching (RIBE) of GaAs in the presence of energetic Ar ions and thermal $\mathrm{O}$ atoms. It includes the collisional effects, diffusive processes and chemical reactions taking place in the system. The model parameters are fitted using the experimental observations of Grigonis [1] and validated with the experimental results obtained during the GaAs ion etching presented in this paper. A detailed analysis is presented to understand the effect of the diffusive processes and the role of $\mathrm{O}$ during RIBE of GaAs. It is shown how the presence of damage caused by the energetic Ar coupled with the presence of thermal O opens up chemical reaction channels which eventually leads to the preferential sputtering of Ga observed at the ion etching facility at University of Greifswald.

* Corresponding author: Abha Rai (Abha.Rai@ipp.mpg.de)

Corresponding author address: Max-Planck-Institute of Plasmaphysics, Wendelsteinstrasse

1, D-17491 Greifswald, Germany.

keywords: GaAs, SDTrimSP, sputtering, etching
\end{abstract}

\section{Introduction}

Dry etching of GaAs is used for the fabrication of nano-meter scale structures such as quantum wires and dots. An energetic ion beam (e. g. Ar) is often applied where the 
energy of the individual ions is used to ablate a target. Introduction of oxygen into the chamber for etching, usually termed as reactive ion beam etching, leads to the formation of oxide layers at the surface of the GaAs target. The oxides act as inhibitors and hinder or even completely prevent the access of etchant to the target atoms. This is useful in the sense that it decreases the side wall etching during the preparation of microstructures.

A simple phenomenological model had been previously reported by Grigonis [1] where they present a set of rate equations for the reactive ion etching of GaAs in $\mathrm{CF}_{2} \mathrm{Cl}_{2}+\mathrm{O}_{2}$ and $\mathrm{O}_{2}$ plasma. The sample was sliced in different layers and the model applied different set of equations for the surface and the deeper layers. The rate equations described the time evolution of the concentrations of various species in different layers. The reaction rate constants were parametrized for a particular set of physical parameters e.g. ion beam energy $(200 \mathrm{eV})$ and substrate temperature $(473 \mathrm{~K})$. Since the diffusion process plays a very important part during the etching processes it is very important to include the effect of the incoming ion beam energy, which due to the damage production leads to different diffusion rates and eventually affects the reaction rates.

The goal of the present work is to include a chemistry module in SDTrimSP version 5.0 for simulating the interaction of GaAs with Ar ions in the presence of thermal oxygen. SDTrimSP [2-4] is a binary collision code which simulates the interaction of energetic particles (e.g. Ar) with a target (e.g. GaAs) taking into account the dynamical changes within the target due to the bombardment and calculates various physical quantities like sputtering yield and depth profiles of different species. The model developed in the present work includes the dynamical effects of the incoming ion beam.

Two experimental scenarios are considered in the present work.

- case 1: The experimental data and the model predictions of Grigonis et al. (for details of experimental set-up see $[1,5])$ and

- case 2: The experimental observation of the preferential sputtering of GaAs during reactive ion beam etching reported in this paper. 
The parameters used in the model are fitted to the results of case 1 . This model is then used to reproduce the experimental observation of case 2. The paper is organized as follows: first the experimental set-up used in case 2 is presented, then the dynamic model developed in the present work is described. This is followed by the results for both of the cases studied and finally a brief summary of the work is presented.

\section{Experimental set-up}

Fig. 1 shows the schematic of the experimental set-up at the University of Greifswald. Energetic $\mathrm{Ar}^{+}$ions are produced by a cold Penning ion source and are accelerated by a $400 \mathrm{kV}$ ion accelerator after mass filtering using an analyzing magnet. There is a beam position monitor and quadrupole lenses to focus the ion beam. The ion beam enters the experimental target chamber by an orifice which is $0.3 \mathrm{~cm}$ in diameter. The target, undoped and chemically polished GaAs (100), is mounted inside a vacuum chamber on a low-cost $\mathrm{x}-\mathrm{y}-\mathrm{z}$ manipulator with the $\mathrm{z}-$ direction pointing along the direction of the incident projectile [6]. An independent $\phi$-rotation around the (vertical) y-axis is also provided and the target can be rotated in such a way that the angle of ion incidence can be changed. In the present study, the angle between the ion beam and the target surface normal is $22.5^{\circ}$. The vacuum chamber is maintained at a base pressure of $8 \times 10^{-9}$ mbar and the target is kept unbiased during the experiment. The ion beam current is kept constant at $0.5 \mu \mathrm{A}$. The analyser is a EQS (Electrostatic Quadrupole SIMS Mass Spectrometer, series 1000) Mass-Energy analyser produced by Hiden Analytical [7,8]. It is positioned at $45^{\circ}$ in plane to the ion beam and $30^{\circ}$ out of plane. The neutral and singly charged positive and negative sputtered species are observed and analysed using the EQS detector. The experiments were carried out at room temperature. 


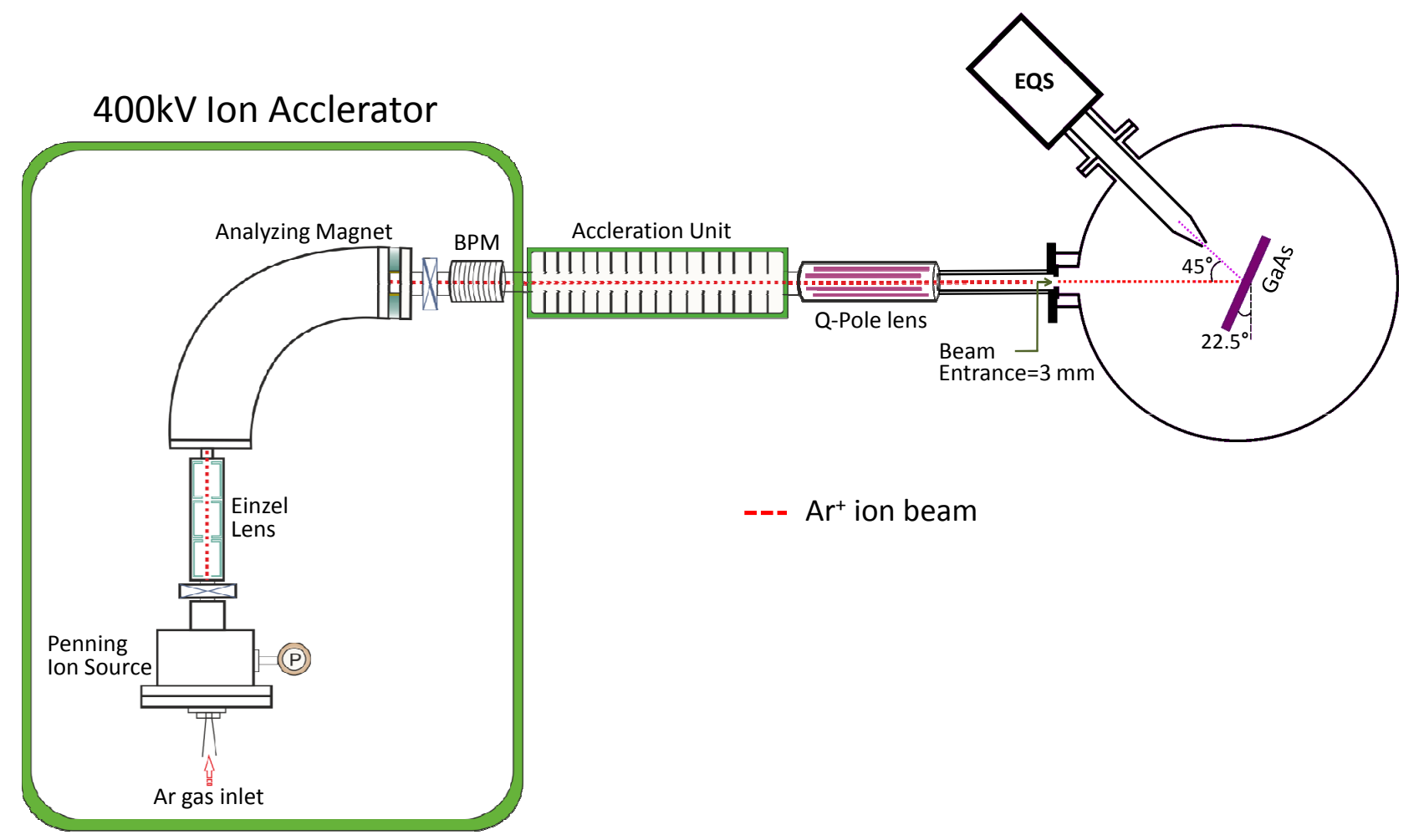

Fig. 1. Scheme of experiment.

\section{Description of model}

Various processes taking place during the reactive ion etching of GaAs are: sputtering, adsorption, desorption, radiation enhanced diffusion and heterogeneous chemical reactions. Depending on the physics behind all these processes, they can be placed under one of the following categories

- collisional processes

- diffusive processes due to fluence dependent damage

- ordinary time dependent diffusive processes

- chemistry related processes and

- temperature dependent desorption processes.

Now all these processes are described in detail. The species considered in the simulation are $\mathrm{Ga}, \mathrm{As}, \mathrm{Ga}_{2} \mathrm{O}_{3}, \mathrm{As}_{2} \mathrm{O}_{3}$, and $\mathrm{O}$ corresponding to specie index $j=1, . ., 5$, respectively. If a sample is bombarded with a flux $\Psi$ and fluence $\Delta \Phi$ per fluence step then the time 
step is $\Delta t=\Delta \Phi / \Psi$. The probability of the change of number of atoms in a layer is the sum of collisional (Ncoll), diffusive (Nddif, Ntdif), chemical process (Nchem) and the temperature dependent process $($ Ntemp) :

$$
\frac{d N}{d \Phi}=N \text { coll }+N \text { ddif }+(N t d i f+N \text { chem }+N \text { temp }) / \Psi
$$

In SDTrimSP program the target is divided into $m$ layers along the $\mathrm{z}$-direction, therefore, any quantity e.g., concentration $\mathrm{c}(\mathrm{z})$ is written as $\mathrm{c}(\mathrm{m})$ in its discrete representation. The absolute number of atoms of type $j$ in a layer $m$ at fluence step $n\left(N_{j, m}^{n}\right)$ is normalized to the area $(\Delta x \cdot \Delta y)$ of the layer, i.e., it is equivalent to the areal density of the atoms. Now each of the terms are described one by one.

\section{Ncoll:}

The number of implanted atoms $n_{i m}$, the number of recoils transferred into $n_{i n}$ and the number of atoms removed $n_{\text {out }}$ from the layer $m$ are given or calculated after the bombardment of target with $n_{r}$ pseudo-particles. Due to the deposition or loss of particles the composition of target is changed. The probability that one incident particle changes the number of atoms of type $j$ in one layer is $N$ coll :

$$
\operatorname{Ncoll}_{j, m}=\frac{n_{\text {im }}(j, m)+n_{\text {in }}(j, m)-n_{\text {out }}(j, m)}{n_{r}} \quad \mathrm{j}=1 \ldots \mathrm{n} \quad \text { species of particle }
$$

Since $N$ coll represents a probability, it is dimensionless.

\section{Nddif:}

$N d d i f$ represents the probability of fluence dependent diffusion coefficient of free-moving particle of specie $j$ in layer $m$. Since fluence also affects the damage caused to a sample, it is damage driven, fluence dependent diffusion. The version 5.0 of SDTrimSP already includes this effect. During the bombardment the implanted Ar atoms lead to a swelling of the target, so that a pressure is built up in the target and it is gradually removed through relaxation. This pressure is described by a concentration gradient term in the target (similar to the diffusion pressure). The implanted Ar atoms are not bound to the 
target and if a pressure gradient exist and the atoms get an external excitation, they move in the direction of the surface (out-gassing). This excitation can be caused by the atomic damages during collisional cascade. The effect of collisional cascade (atomic damages) starts at the surface and end deep in the target, there the diffusion of the atoms is only possible in the direction of the surface. This effect is described mathematically by the following classical diffusion like equation.

$$
N d d i f_{j, m}=\frac{d\left[\eta_{d}(j, m) \cdot \frac{c_{j, m}}{d z}\right]}{d z} \cdot \Delta z_{m}^{n}
$$

with the concentration $c_{j, m}=f_{j, m} \cdot \varrho_{m}$, where $f_{j, m}$ is the atomic fraction and $\varrho_{m}$ is the mean atomic density of layer $m$. Here $\Delta z_{m}^{n}$ is the thickness of layer $m$ at the fluence step $n$. In SDTrimSP the geometrical surface is assumed to be at $z=0$ and by this convention depth of the sample along the $\mathrm{z}^{-}$-axis (also $\Delta z$ ) is negative. Therefore, diffusion equations like eqn. 3, have a positive sign unlike the classical diffusion equation which has a negative sign on the right hand side. In the present work this convention is implied in all the diffusion equations. Note that unlike classical time-dependent the diffusion coefficient (dimension: $(\text { length })^{2} /($ time $)$, units: $\left.m^{2} s^{-1}\right), \eta_{d}(z, j)$ is a variable determined locally on the fluence driven damage (dimension: $(\text { lengt } h)^{2} /\left(\right.$ fluence), units: $\left.m^{4}\right)$. The diffusion coefficient $\eta_{d}(j, m)$ is dependent on a constant diffusion coefficient $\eta_{d 0}$, the dimensionless damage profile $P_{d a m}$ and the atomic fraction $f$ of species $\mathrm{j}$.

$$
\begin{aligned}
& \eta_{d}(j, m)=\eta_{d 0}(j) \cdot P_{d a m}(m) \cdot f_{j, m} \\
& P_{d a m}(m)=\frac{n_{\text {out }}(m)}{\max \left(n_{\text {out }}\right)}
\end{aligned}
$$

Here $n_{\text {out }}$ is the number of atoms removed from the layer $m$ and $\max \left(n_{\text {out }}\right)$ is the maximum of $n_{\text {out }}$ from all the layers. Since $\mathrm{O}$ is a gas, $\mathrm{O}$ atoms diffuse freely in the collision cascade induced damage region of the target. Therefore the damage driven diffusion is used only for oxygen. The parameter $\eta_{d 0}$ was fitted to the profiles observed in case 1 and the values used in the present work is given in table 1. 


\section{Ntdif:}

GaAs lattice has many vacancies of As because of its high chemical activity and low sublimation energy. These vacancies are occupied by the oxygen atoms from the plasma and eventually leading to the process of inter-diffusion of As and O. A detailed atomistic analysis has been carried out by Taguchi et al. for calculating the activation energy barrier of $\mathrm{O}$ in GaAs [9]. Ntdif represents the time-dependent diffusive flux and $N t \operatorname{dif} / \Psi$ is the probability of such events. It includes standard diffusion with a constant diffusioncoefficient $\eta_{0}(j)$.

$$
N t d i f_{j, m}=\eta_{0} \frac{d^{2} c_{j, m}}{d z^{2}} \cdot \Delta z_{j, m}^{n}
$$

The diffusion term $N$ tdif for arsenic $(\mathrm{j}=2)$ at the time step $\mathrm{n}$ is:

$$
N t d i f_{2, m}^{n}=\eta_{0}(A s) \cdot \frac{d^{2}\left(f_{2, m} \cdot \varrho_{m}\right)}{d z^{2}} \cdot \Delta z_{m}^{n}
$$

The value of $\eta_{0}(A s)$ is given in table 1 and this value corresponds to the diffusion coefficient of As in the bulk of GaAs. There is a finite probability that some of As atoms will leave the surface. Therefore, the flux of As diffusing out of the surface has to be limited. The physics basis for this is the fact that the surface binding energy of GaAs is of the same order as the activation energy for the diffusion of As (in the range of $3-4 \mathrm{eV}$ ) $[9,10]$. This is reflected in the SDTrimSP simulations by using a reduced value of the diffusion coefficient $\eta_{0}(A s)$ at the surface. The best fit of the profiles of different species for case 1 are obtained when the $\eta_{0}(A s)$ at the surface is taken as $10 \%$ of the bulk value of $\eta_{0}(A s)$.

For O there are two terms contributing to Ntdif see [1]. The inter-diffusion (arsenic \& oxygen) and diffusion term $N$ tdif for oxygen $(\mathrm{j}=5)$ is:

$$
N t d i f_{5, m}^{n}=\eta_{0}(O) \cdot \frac{d^{2}\left(f_{5, m} \cdot \varrho_{m}\right)}{d z^{2}} \cdot \Delta z_{m}^{n}-N t d i f_{2, m}^{n}
$$

The first term on the right hand side comes from the thermal diffusion of $\mathrm{O}$ given by the constant diffusion-coefficient $\eta_{0}(O)$. The second term is due to the anti-diffusion of 
O, replacing the out-diffusing As. The is implemented by doing the flux balance of outdiffusing As and O. The out-diffusing flux of As from a layer is calculated and then the equal amount of $\mathrm{O}$ is allowed to diffuse into the given layer.

\section{Nchem:}

The chemical reaction included in the model is basically oxidation, i.e., formation of $\mathrm{Ga}_{2} \mathrm{O}_{3}$ and $\mathrm{As}_{2} \mathrm{O}_{3}$, and they are represented by the following rate equations. The chemical terms Nchem [1] for five species are:

$$
\begin{aligned}
& \operatorname{Nchem}_{1, m}^{n}=-2 \cdot R_{1} \cdot \varrho_{m}^{n} \cdot \Delta z_{m}^{n} \\
& \operatorname{Nchem}_{2, m}^{n}=-2 \cdot R_{2} \cdot \varrho_{m}^{n} \cdot \Delta z_{m}^{n} \\
& \operatorname{Nchem}_{3, m}^{n}=+1 \cdot R_{1} \cdot \varrho_{m}^{n} \cdot \Delta z_{m}^{n} \\
& \operatorname{Nchem}_{4, m}^{n}=+1 \cdot R_{2} \cdot \varrho_{m}^{n} \cdot \Delta z_{m}^{n} \\
& \operatorname{Nchem}_{5, m}^{n}=-3 \cdot\left(R_{1}+R_{2}\right) \cdot \varrho_{m}^{n} \cdot \Delta z_{m}^{n}
\end{aligned}
$$

$N c h e m$ is the reaction rate per unit area and $N c h e m / \Psi$ gives the probability of an chemical reaction event. The reaction rate of Ga and As with oxygen is $R_{1}$ and $R_{2}$, respectively, and are given by.

$$
\begin{aligned}
& R_{1}=k_{1, m} \cdot\left(f_{m, 1}^{n}\right)^{2} \cdot\left(f_{m, 5}^{n}\right)^{3} \quad \text { due to }:\left(2 G a+3 O->G a_{2} O_{3}\right) \\
& R_{2}=k_{2, m} \cdot\left(f_{m, 2}^{n}\right)^{2} \cdot\left(f_{m, 5}^{n}\right)^{3} \quad \text { due to }:\left(2 A s+3 O->A s_{2} O_{3}\right)
\end{aligned}
$$

It should be noted that Grigonis et al. used different values of $R_{1}$ and $R_{2}$ for surface (first monolayer) and bulk $\left(k_{m=1} \neq k_{m>1}\right)$, see table 2 .

\section{Ntemp:}


Ntemp represents the desorbed out-flux of $\mathrm{As}_{2} \mathrm{O}_{3}$ at the surface. It is temperature dependent and is given by

$$
N t e m p_{4, m}^{n}=\nu \cdot e^{-E d / k T} \cdot \varrho_{m}^{n} \cdot \Delta z_{m}^{n}
$$

Ntemp/ $\Psi$ gives the probability of particle removal from the surface due to desorption. The frequency probability of particle removal from the surface as a result of desorption is given by $\omega_{D}=\nu \cdot e^{-E d / k T}$, where $E d$ is the desorption energy, $k$ is the Boltzmann's constant, $T$ is the temperature and $\nu$ is the attempt frequency for desorption process, it is taken typically as the frequency of atom oscillation in a lattice $\left(\sim 10^{12} s^{-1}\right)$. For $\mathrm{As}_{2} \mathrm{O}_{3}$ $\omega_{D}=0.05 \mathrm{~s}^{-1}$ at $200^{\circ} \mathrm{C}([1])$, this implies that $E d=1.25 \mathrm{eV}$.

\begin{tabular}{|r|c|c|c|c} 
nr. & species & $\varrho_{0}$ & $\begin{array}{c}\eta_{d 0} \\
{\left[\AA^{4} / \AA^{-3}\right]}\end{array}$ & $\begin{array}{c}\eta_{0} \\
{\left[\AA^{2} / \text { atom }\right]}\end{array}$ \\
\hline 1 & $G a$ & 0.05099 & - & - \\
2 & $A s$ & 0.04603 & - & 240 \\
3 & $\mathrm{Ga}_{2} \mathrm{O}_{3}$ & 0.01889 & - & - \\
4 & $\mathrm{As}_{2} \mathrm{O}_{3}$ & 0.01127 & - & - \\
5 & $O$ & 0.04291 & 400 & 550
\end{tabular}

Table 1

The values of atomic density $\left(\varrho_{0},[4]\right)$, fluence-dependent and time-dependent diffusion coefficients for various species.

\begin{tabular}{|r|c|c|}
$\begin{array}{r}\text { Rate } \\
\text { const. }\end{array}$ & $\begin{array}{c}\text { Grigonis } \\
\text { et al. }\end{array}$ & $\begin{array}{c}\text { Present } \\
\text { work }\end{array}$ \\
\hline$k_{1}$ & $1.000^{(m=1)}, 0.167^{(m>1)}$ & 0.045 \\
$k_{2}$ & $0.570^{(m=1)}, 0.096^{(m>1)}$ & 0.300
\end{tabular}

Table 2

Chemical reaction rate constant $k_{1}$ for Ga and $k_{2}$ for As (in units of $s^{-1}$ ). 
After using all the terms described above in eqn. 1 and applying a simple Euler's method the time evolution of different species is calculated. Let $n$ represent the time or fluence step index, then eqn. 1 can be written in the discrete form as

$$
\begin{aligned}
N_{j, m}^{n+1}= & N_{j, m}^{n}+\left(\text { Ncoll }_{j, m}^{n}+N \operatorname{Ndif}_{j, m}^{n}\right) \cdot \Delta \Phi \\
& +\left(N t d i f_{j, m}^{n}+N \operatorname{chem}_{j, m}^{n}+N t e m p_{j, m}^{n}\right) \cdot \Delta t
\end{aligned}
$$

If $f_{j, m}^{n}$ denotes the atomic fraction, $\Delta z_{m}^{n}$ the thickness, $\varrho_{m}^{n}$ the mean atomic density , and $N_{j, m}^{n}\left(=f_{j, m}^{n} \cdot \varrho_{m}^{n} \cdot \Delta z_{m}^{n}\right)$ the areal number density of layer $m$ at fluence step $n$, then

$$
\begin{aligned}
N_{j, m}^{n+1}= & f_{j, m}^{n} \cdot \varrho_{m}^{n} \cdot \Delta z_{m}^{n}+\left(\operatorname{Ncoll}_{j, m}^{n}+N d d i f_{j, m}^{n}\right) \cdot \Delta \Phi \\
& +\left(N t d i f_{j, m}^{n}+N \operatorname{chem}_{j, m}^{n}+N t e m p_{j, m}^{n}\right) \cdot \frac{\Delta \Phi}{\Psi}
\end{aligned}
$$

The new thickness $z^{n+1}$ of the layer $m$ after one fluence step $\Delta \Phi$ can be calculated:

$$
\Delta z_{m}^{n+1}=\sum_{j} \frac{N_{j, m}^{n+1}}{\varrho_{0}(j)}
$$

The new composition or new the atomic fraction $f^{n+1}$ is:

$$
f_{j, m}^{n+1}=N_{j, m}^{n+1} / \sum_{k} N_{k, m}^{n+1} \quad j, k \quad \text { species of particle }
$$

The new mean atomic density $\varrho_{m}$ is:

$$
\frac{1}{\varrho_{m}^{n+1}}=\sum_{j} \frac{f_{j, m}^{n+1}}{\varrho_{0}(j)}
$$

\section{Results}

As discussed above the whole physics scenario of the ion-beam etching of GaAs can be splitted into the contribution from the collisional processes (Ncoll), diffusive processes due to fluence dependent damage (Nddif), ordinary time dependent diffusive processes (Ntdif), 
chemistry related processes (Nchem) and temperature dependent desorption processes (Ntemp). In order to understand the detailed dynamics and the contribution of each term to the overall outcome separately, they are activated step by step (except Ncoll and Ntemp, which are always active).

\subsection{Case 1:}

Fig. 2 shows the contribution of each term to the overall profiles of the various species. In fig. 2a binary collisional (Ncoll), damage induced diffusion (Nddif) and thermal effects (Ntemp) are taken into account. This gives bad agreement between the experimental and the model predictions, emphasizing the importance of Nchem and Ntdif terms. Inclusion of inter-diffusion of As and $\mathrm{O}$ already gives a better agreement for the As and $\mathrm{O}$ profiles, indicating that inter-diffusion of As/O plays the most crucial role for their profiles (see Fig. 2b). It is also seen that the inclusion of inter-diffusion has practically no effect on the profile of the Ga. Fig. 2c shows the outcome due to further inclusion of Ntdif of O (i.e. the time dependent constant diffusion of $\mathrm{O}$ ). Note that it is this process which leads to the free, constant diffusion of $\mathrm{O}$ leading to the reactions and oxide formation of $\mathrm{Ga}$ and therefore it has a lot of influence on the profile of elemental Ga. The inclusion of Ntdif of O leads to the good agreement of the Ga profile with experiment. Fig. 2d shows

the profile of all the species (including oxides) considered in the simulation and it agrees very well with the experimental data (only elemental profiles) and the phenomenological model prediction by Grigonis et al.

Fig. 3 shows the time development (or equivalently fluence dependence in the present scheme) of the profile of various species. Grigonis et al. show that the system is very dynamic and the exact composition of the layer keeps changing. The results reported by them are the measurements at a given instant of time. Nevertheless, it is clearly seen in fig. 2 that the surface layer is depleted by As and enriched by Ga as observed in the experiment (Fig. 2 [5]). This is due to the inter-diffusion of oxygen where As segregates to the surface and their sites are occupied by the adsorbed $\mathrm{O}$ atoms. The altered sample 
(a)

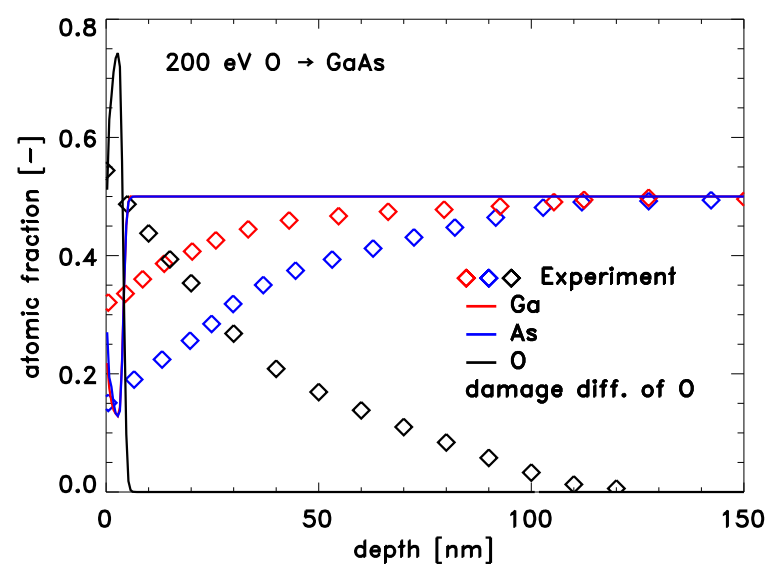

(c)

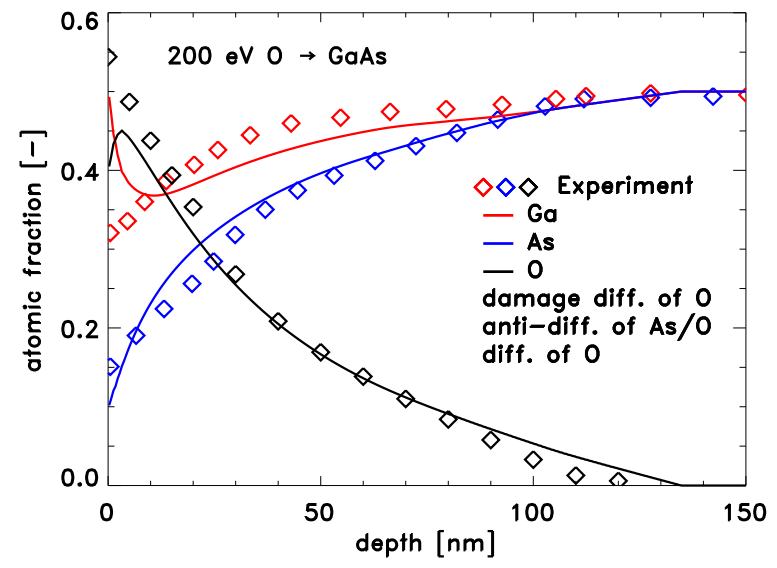

(b)

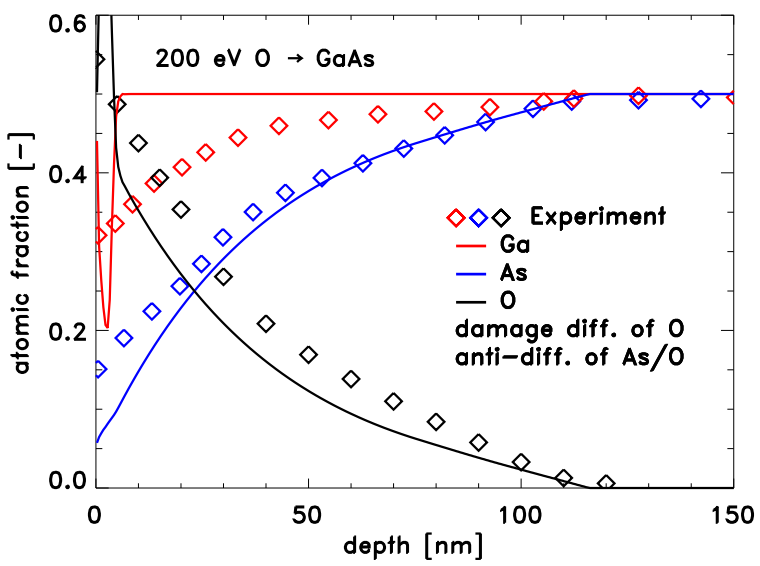

(d)

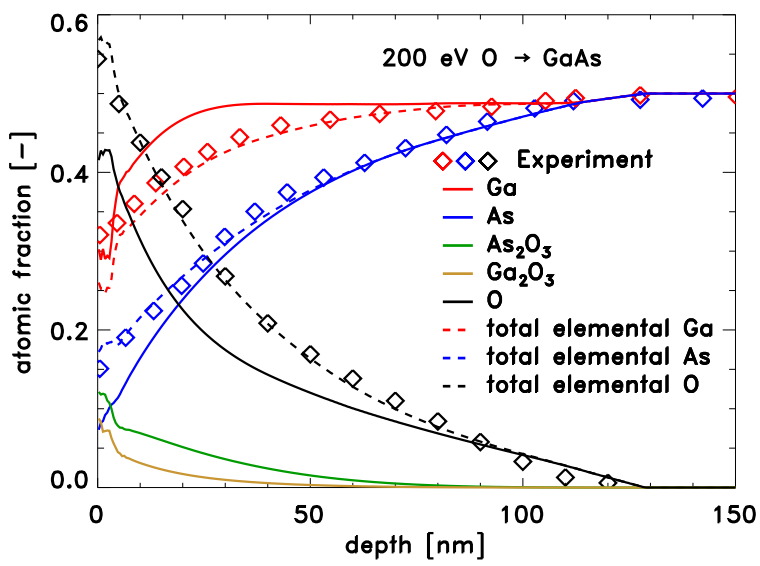

Fig. 2. Comparison of the calculated depth profiles of the atomic fraction for various species (a) only Nddif of $\mathrm{O}$ (b) Nddif of $\mathrm{O}$ and only inter-diffusion of As/O contribution from Ntdif (c) Nddif of $\mathrm{O}$ and full Ntdif term (d) all processes/terms included (Nchem as well).

also contains oxides of Ga and As.

Fig. 4 shows the dependence of the oxide layer thickness on the current density of Ar ions. The model calculations are compared with the experimental data (Fig. 3 [1]) and a very good agreement is observed between the two.

Fig. 5 shows the temperature dependence of the profiles of various species. It is clearly indicated (as also observed in experiments) that temperature is also a very important parameter affecting the composition of the altered layer. However, in the present work the direct temperature dependence is only included through Ntemp term. But the authors 


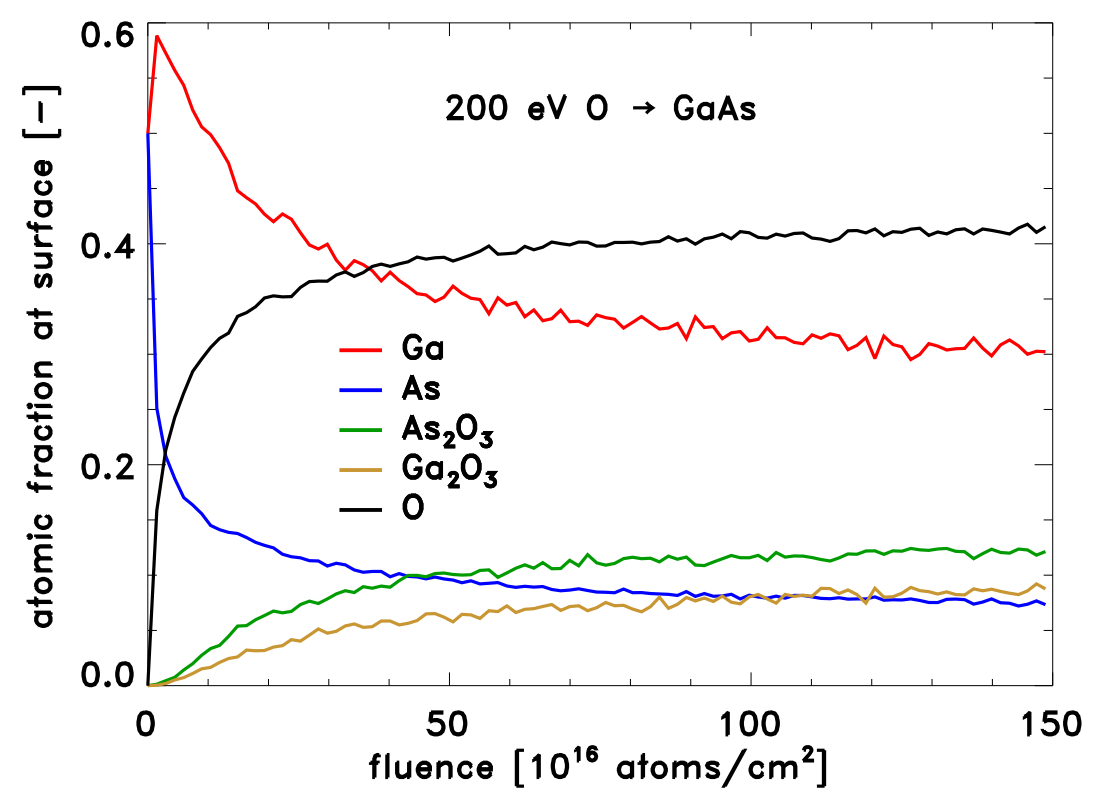

Fig. 3. Time development of the profiles of various species at the surface layer for $200 \mathrm{eV} \mathrm{O}$ on GaAs target.

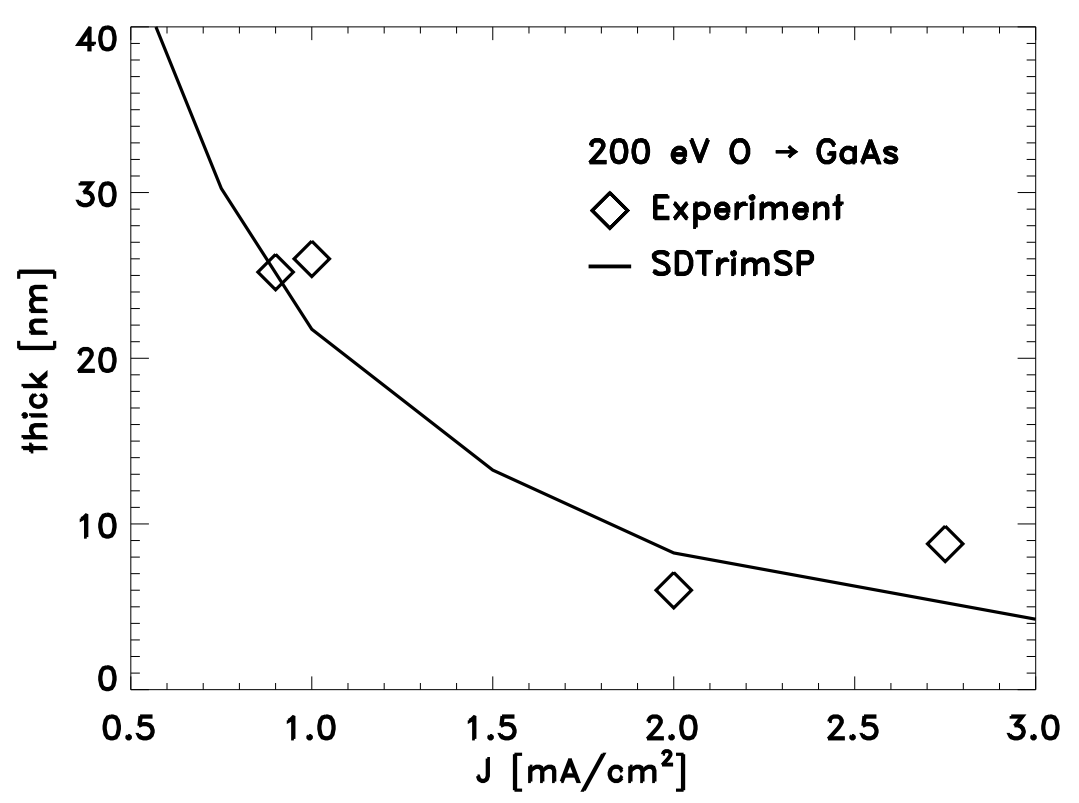

Fig. 4. The dependence of the thickness of the oxide layer as a function of current density for $200 \mathrm{eV} \mathrm{O}$ on GaAs target.

are fully aware that other terms, specially Nchem is strongly temperature dependent and an upgraded model including this dependence globally and self-consistently is needed to 
explore the temperature dependence.

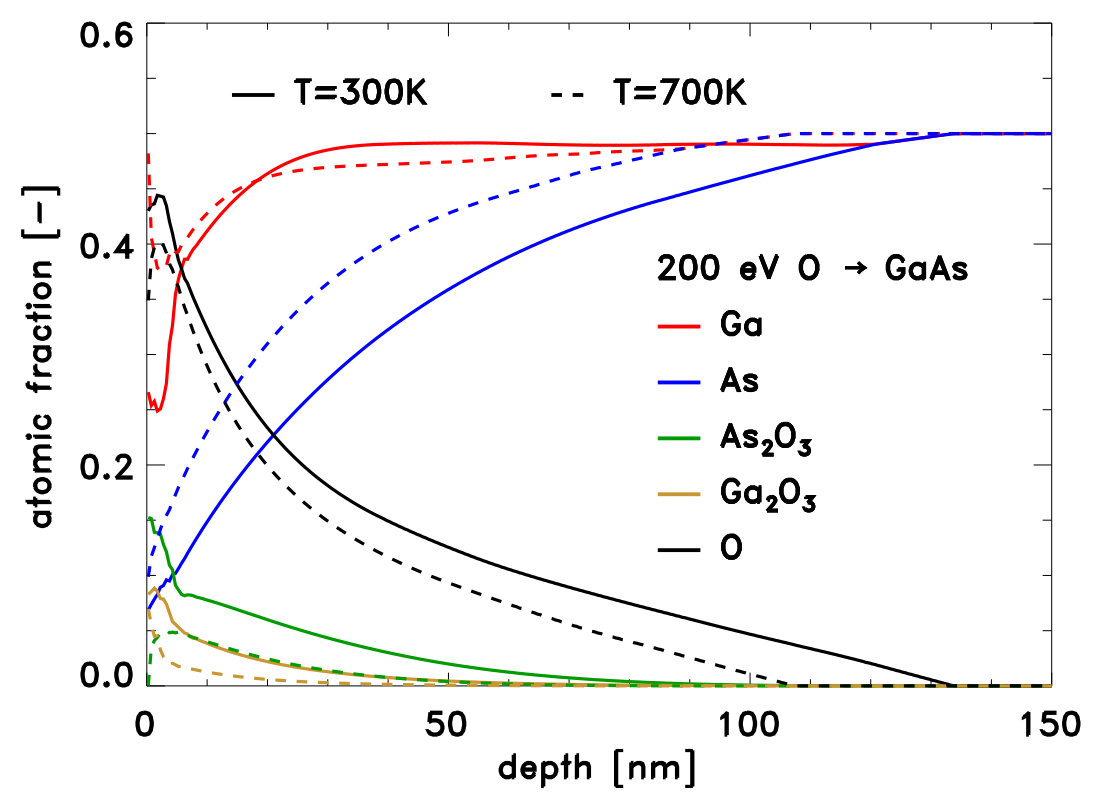

Fig. 5. Temperature dependence of the profile of various species.

\subsection{Case 2:}

Fig. 6 shows the experimental data obtained during the sputtering of a GaAs target by $150 \mathrm{keV} \mathrm{Ar}^{+}$ions in the presence of thermal $\mathrm{O}_{2}$ molecules at room temperature. Preferential sputtering of $\mathrm{Ga}^{+}$ions compared to $\mathrm{As}^{+}$is clearly observed. The measured energy dependencies are rather similar for $\mathrm{Ga}^{+}$and $\mathrm{As}^{+}$and drop off close to an $\mathrm{E}^{-2}$ dependency. In addition, sputtering of gallium and arsenic oxides, e.g., $\mathrm{GaO}^{+}, \mathrm{GaO}_{2}^{+}$, and $\mathrm{AsO}^{+}$is noted. The measured energy dependencies show a clear tendency to become steeper with increasing number of atoms per sputtered molecule.

Various SDTrim calculations were performed to study the impact of $\mathrm{O}$ and diffusion of As on the total sputtering yield of $\mathrm{Ga}$ and As, their outcome is listed in table 3 and is discussed below. The ejected flux is splitted in terms of the contribution coming from the physical sputtering and the diffusion of the species. Even though both As and O can diffuse freely in the target, the diffusive flux is non-zero only for As, because only this 


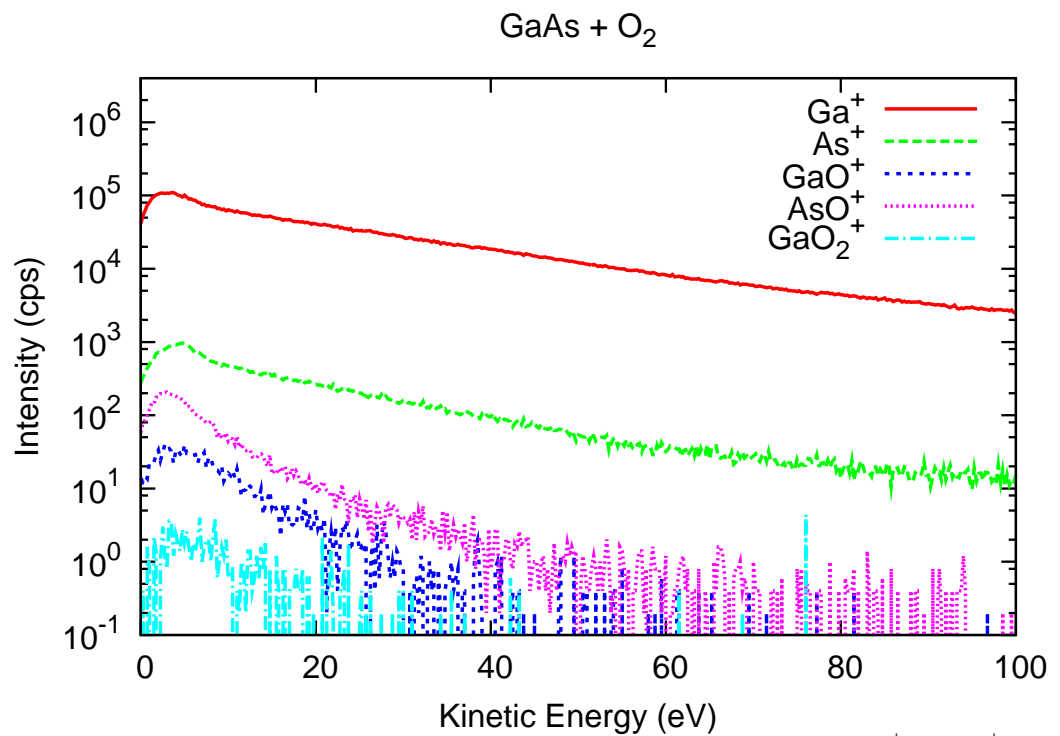

Fig. 6. Measured energy dependence of sputtered $\mathrm{Ga}^{+}, \mathrm{As}^{+}, \mathrm{GaO}^{+}, \mathrm{AsO}^{+}$, and $\mathrm{GaO}_{2}^{+}$ions following $150 \mathrm{keV} \mathrm{Ar}^{+}$ion bombardment of a GaAs target in the presence of oxygen $\left(\mathrm{O}_{2}\right.$, partial pressure $2 \times 10^{-6}$ mbar).

specie is diffusing out of the surface. $\mathrm{O}$ is diffusing into the target (see Fig. 2, $\mathrm{O}$ and As have almost inverse profiles).

(1) $\mathrm{Ar}^{+} \rightarrow$ GaAs (without diffusion of As and O): Fig. 7(a), as expected, using the simple collisional cascade model, due to the similar masses of Ga and As, the sputtering yield of $\mathrm{Ga}$ and As were equal.

(2) $\mathrm{Ar}^{+} \rightarrow$ GaAs (no oxygen, with diffusion of As): Fig. 7(b), preferential sputtering of Ga is observed. The sputtering yield of Ga and As were 2.096 and 0.653 , respectively. The diffusion coefficient of As is much higher than that of Ga [11]. Due to the higher diffusion coefficient of As the range of depletion is larger than without diffusion as discussed for case 1. This leads to fewer As atoms close to the surface layers which can be sputtered. This result matches very well with the observation of Mahlerbe et al. [12] where they observed the preferential sputtering of $\mathrm{Ar}^{+} \rightarrow \mathrm{GaAs}$ (without O). It must be noted that the diffusive flux has much higher contribution to the total ejected As indicating that diffusion of As is a dominant process.

(3) $\mathrm{Ar}^{+}+\mathrm{O} \rightarrow \mathrm{GaAs}$ (with diffusion of $\mathrm{O}$ only): Fig. 7(c), in the absence of diffusion of As and anti-diffusion of $\mathrm{O}$ the sputtering yield of $\mathrm{Ga}$ and As were 0.354 and 0.153 , respectively. Preferential sputtering of Ga is observed but not as much as in the case where diffusion of As is taken into account. 
(4) $\mathrm{Ar}^{+}+\mathrm{O} \rightarrow \mathrm{GaAs}$ (including all the processes): Fig. 7(d), the sputtering yield of Ga and As were 0.393 and 0.075 , respectively. The ratio of total sputtered flux of Ga/As is 5.2. It can be seen from Fig. 7(b) and Fig. 7(c) that the diffusion of As gives the higher contribution to the preferential sputtering of Ga (Fig. 7(b),Ga/As ratio 3.2) than the chemical processes related to the diffusion of O only (Fig. 7(c), Ga/As ratio 2.3). It also indicates that second term of Ntdif for O (Eq. 8), corresponding to the anti-diffusion is dominant compared to the thermal diffusion of $\mathrm{O}$ (first term using $\left.\eta_{0}(O)\right)$.

(a)

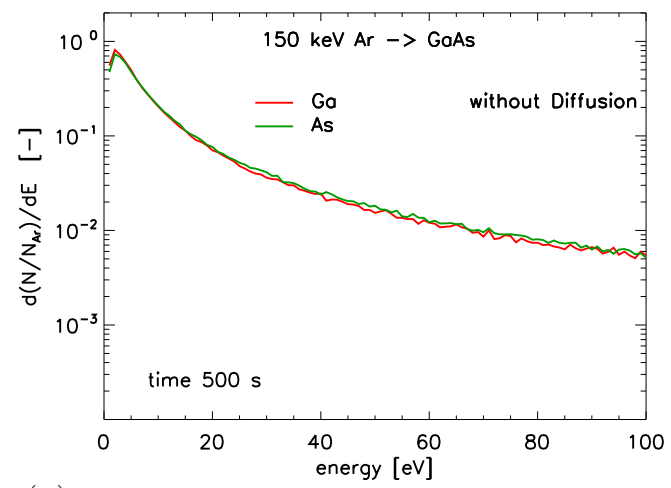

(c)

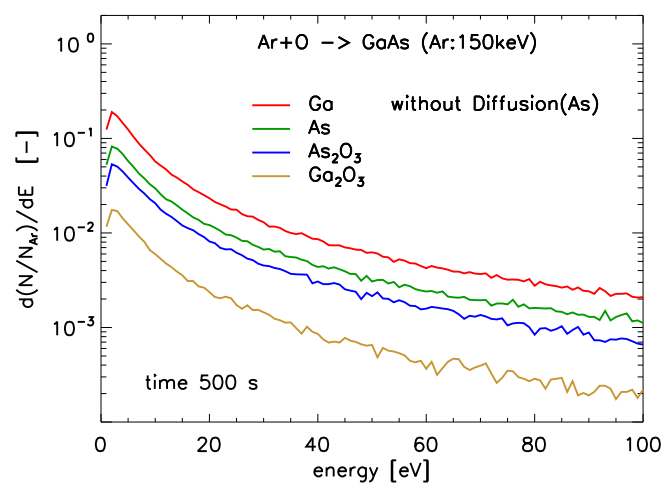

(b)

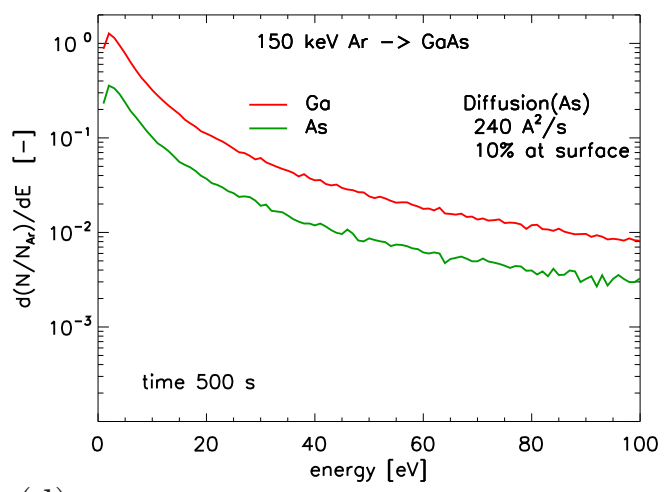

(d)

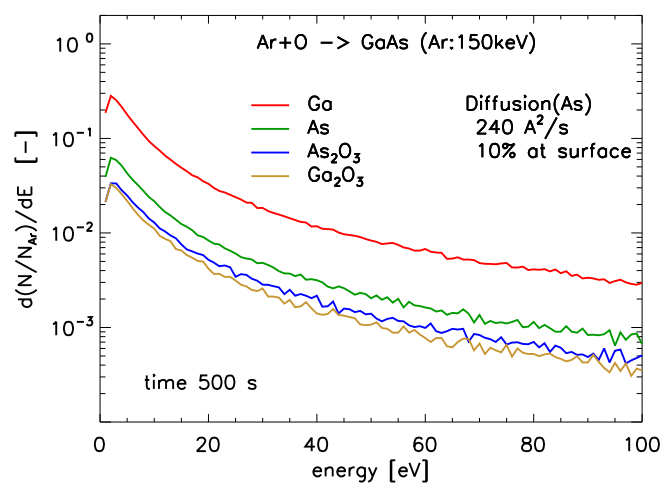

Fig. 7. Energy dependence of the normalized flux of sputtered species.(a) $A r \rightarrow G a A s$, pure collisional cascade without diffusion, (b) $A r \rightarrow G a A s$, including diffusion of As, (c) $A r+O \rightarrow G a A s$, without diffusion of As (therefore, no anti-diffusion of $\mathrm{O}$ ) and (d) $\mathrm{Ar}+\mathrm{O} \rightarrow \mathrm{GaAs}$, including all the processes.

With all the scenarios presented above, it can be concluded that the diffusion of As reduces its amount in the surface layers. This gives rise to a Ga rich surface layer and the preferred 


\begin{tabular}{|r|cl|rccc|c|} 
Figure & Total Sputtering Yield & \multicolumn{3}{|c|}{ Sputtered Flux } & Diffusive Flux \\
number & $\mathrm{Y}(\mathrm{Ga}) \mathrm{Y}(\mathrm{As})$ & $Y_{S}\left(\mathrm{Ga}, \mathrm{As}, \mathrm{Ga}_{2} \mathrm{O}_{3}, \mathrm{As}_{2} \mathrm{O}_{3}\right)$ & $Y_{D}(\mathrm{As})$ \\
\hline $7(\mathrm{a})$ & 1.403 & 1.403 & 1.0 & 1.0 & 0.0 & 0.0 & 0.0 \\
$7(\mathrm{~b})$ & 2.096 & 0.653 & 1.0 & 0.316 & 0.0 & 0.0 & 0.684 \\
$7(\mathrm{c})$ & 0.354 & 0.153 & 0.841 & 0.445 & 0.159 & 0.555 & 0.0 \\
$7(\mathrm{~d})$ & 0.393 & 0.075 & 0.893 & 0.145 & 0.107 & 0.164 & 0.691
\end{tabular}

Table 3

The total sputtering yield, fraction of the sputtered and diffusive fluxes of various species ejected from GaAs target for different scenarios presented in Fig. 7.

sputtering of $\mathrm{Ga}$. In the presence of $\mathrm{O}$, the inter-diffusion of As and $\mathrm{O}$ starts, leading to much higher preferential sputtering of Ga.

Fig. 7(d) shows the modeling result for the case 2 experiment and the relative difference in the sputtered amount of Ga and As is very well reproduced. It must be noted that with the same set of parameters (fitted for case 1 experiment having $200 \mathrm{eV} \mathrm{O}$ and $473 \mathrm{~K}$ target), the presented model is able to reproduce the experimental results of case 2 (150 $\mathrm{keV} \mathrm{Ar}^{+}$and thermal $\mathrm{O}$ on a target at $\left.273 \mathrm{~K}\right)$.

Fig. 8 shows the calculated profile of various species for case 2. As also seen for case 1 , the near surface layer is enriched with Ga and depleted by As. The concentration of $\mathrm{O}$ is very high at the surface and then it falls gradually, As has an almost inverse profile. This can again be understood due to the phenomenon of inter-diffusion of $\mathrm{O}$ and As. In the near-surface layer oxides of Ga and As are observed. Deep into the bulk, both Ga and As are present in the elemental form and very little oxide concentration is observed.

\section{Conclusions}

A SDTrimSP model simulating the dynamics of altered layer formation during the etching process of GaAs target has been presented. The model parameters are fitted using the experimentally measured profiles by Grigonis [1] and are validated by reproducing 


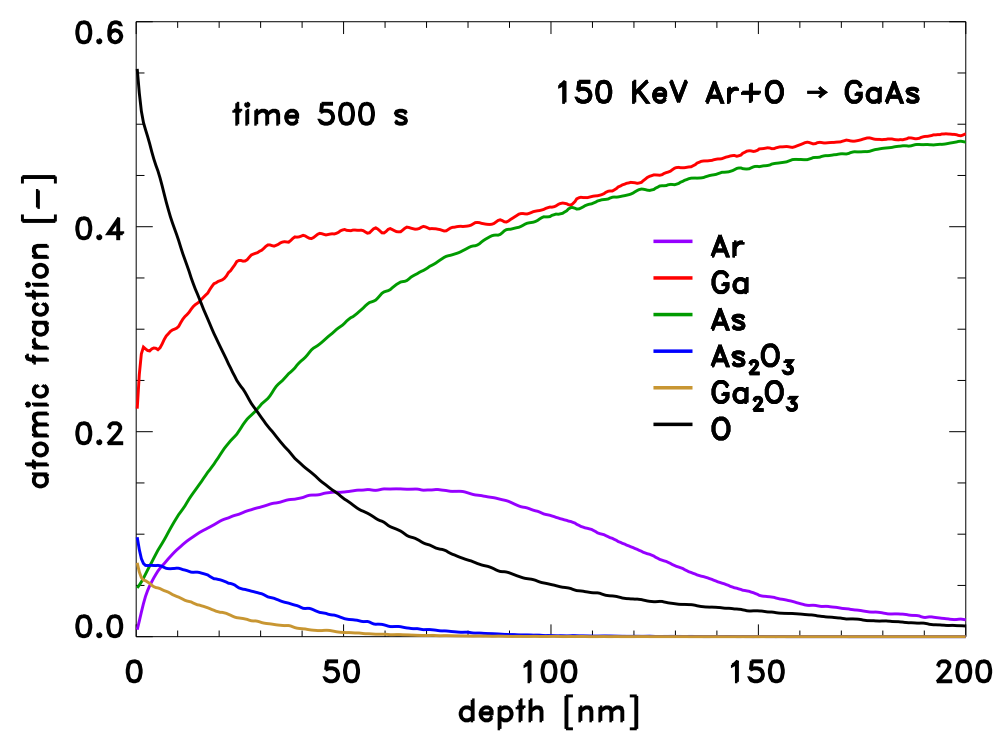

Fig. 8. Calculated profile of atomic fraction of various species for $150 \mathrm{keV}$ Ar on GaAs target with thermal oxygen after $500 \mathrm{~s}$.

the presented results for RIBE of GaAs. Good agreement has been found between the experiments and the model predictions. The sputtering of GaAs by $A r^{+}$both with and without the presence $\mathrm{O}$ is studied. The diffusion of As reduces its amount in the surface layers. This gives rise to Ga rich surface layer and the preferred sputtering of Ga. In the presence of $\mathrm{O}$, the process of anti-diffusion of $\mathrm{O}$ dominates and starts leading to much higher preferential sputtering of Ga. An updated model to represent the overall temperature dependence is needed.

\section{Acknowledgement}

Part of the work was supported by the Deutsche Forschungsgemeinschaft (DFG) through Sonderforschungsbereich SFB/TRR24. One of us (AEJ) is grateful for a grant (Landesgraduiertenstipendium) provided through the University of Greifswald. 


\section{References}

[1] A. Galdikas A. Grigonis and M. Silinskas. Appl. Surf. Sci., 138-139:581-586, (1999).

[2] W. Eckstein A. Mutzke, R. Schneider and R. Dohmen. SDTrimSP: Version 5.00. IPP, Report, (12/8), 2011.

[3] A. Mutzke and W. Eckstein. Nucl. Instr. and Meth. B, 266:872-876, (2008).

[4] W.Eckstein A. Mutzke, R. Schneider and R. Dohmen. SDTrimSP Version 5.00. IPP, Report, $(12 / 8), 2011$.

[5] A. Galdikas A. Grigonis and L. Pranevicius. Vacuum, 51:211-215, (1998).

[6] R. Hippler and Roger Krupinski. Nucl. Inst. and Meth. in Phys. Res. B, 269:3209-3211, (2011).

[7] C.Pagura A.Tolstogouzov, S.Daolio and C.L. Greenwood. International Journal of Mass Sepctrometry, 214:327-337, (2002).

[8] Hiden Analytical Ltd. 420 Europe Boulevard. Warrington WA5 7UN, England.

[9] A. Taguchi and H. Kageshima. Phys. Rev. B, 60(8):5383-5391, (1999).

[10] P. Heimann D. E. Eastman, T. C. Chiang and F. J. Himpsel. Phys. Rev. Lett., 45:656-659, (1980).

[11] J. F. Wager. J. Appl. Phys., 69(5):3022-3031, (1991).

[12] I. Le R. Strydom J. B. Malherbe, W. O. Barnard and C. W. Louw. Surf. and Interface Anal., 18:491-495, (1992). 


\section{List of Figures}

$1 \quad$ Scheme of experiment.

2 Comparison of the calculated depth profiles of the atomic fraction for various species (a) only Nddif of $\mathrm{O}$ (b) Nddif of $\mathrm{O}$ and only inter-diffusion of As/O contribution from Ntdif (c) Nddif of O and full Ntdif term (d) all processes/terms included (Nchem as well).

3 Time development of the profiles of various species at the surface layer for $200 \mathrm{eV}$ O on GaAs target.

4 The dependence of the thickness of the oxide layer as a function of current density for $200 \mathrm{eV} \mathrm{O}$ on GaAs target.

5 Temperature dependence of the profile of various species.

6 Measured energy dependence of sputtered $\mathrm{Ga}^{+}, \mathrm{As}^{+}, \mathrm{GaO}^{+}, \mathrm{AsO}^{+}$, and $\mathrm{GaO}_{2}^{+}$ions following $150 \mathrm{keV} \mathrm{Ar}^{+}$ion bombardment of a GaAs target in the presence of oxygen $\left(\mathrm{O}_{2}\right.$, partial pressure $\left.2 \times 10^{-6} \mathrm{mbar}\right)$.

$7 \quad$ Energy dependence of the normalized flux of sputtered species.(a) $A r \rightarrow G a A s$, pure collisional cascade without diffusion, (b) $A r \rightarrow G a A s$, including diffusion of As, (c) $A r+O \rightarrow G a A s$, without diffusion of As (therefore, no anti-diffusion of $\mathrm{O}$ ) and (d) $\mathrm{Ar}+\mathrm{O} \rightarrow \mathrm{GaAs}$, including all the processes.

8 Calculated profile of atomic fraction of various species for $150 \mathrm{keV}$ Ar on GaAs target with thermal oxygen after $500 \mathrm{~s}$. 